\title{
Effect of experimental temporomandibular disorder pain on sleep bruxism: a pilot study in males
}

\author{
Konstantin Muzalev ${ }^{1}$ (D) $\cdot$ Corine M. Visscher $^{1} \cdot$ Michail Koutris $^{1} \cdot$ Frank Lobbezoo $^{1}$
}

Received: 22 June 2017 / Accepted: 2 April 2019 / Published online: 26 April 2019

(C) The Author(s) 2019

\begin{abstract}
Objectives Sleep bruxism (SB) is considered to play an important role in the provocation of temporomandibular disorder (TMD) pain. However, clinical studies investigating this relation yielded contradictory results. These contradictory results can, at least in part, be explained by a possible influence of TMD pain on SB activity. The aim of this experimental study was to assess the effect of TMD pain on SB.

Materials and methods Nine male participants with clinical signs of SB underwent two subsequent baseline ambulatory polysomnographic (PSG) recordings before undergoing an experimental pain provocation protocol. Thirty-two hours after the pain provocation part a third ambulatory PSG recording was obtained to study the effect of pain on SB.

Results Decrease for all bruxism parameters was found between the recording after the provocation part and the second baseline recording.

Conclusions Experimentally induced TMD pain causes a reduction in SB activity in healthy individuals.

Clinical relevance A reduction in sleep bruxism activity was recorded in all participants who experienced jaw-muscle pain. This is in line with the pain adaptation model. It supports the negative association between sleep bruxism and jaw muscle pain reported by numerous polysomnographic studies.
\end{abstract}

Keywords Bruxism $\cdot$ Temporomandibular disorder $\cdot$ Pain provocation experiment

\section{Introduction}

Bruxism is defined as a repetitive jaw-muscle activity characterized by clenching or grinding of the teeth and/or by bracing or thrusting of the mandible [1]. It has two circadian manifestations occurring either during wakefulness (wake bruxism) or sleep (sleep bruxism). Among other oral parafunctions, sleep bruxism (SB) is considered very common in the general population, with a self-reported prevalence of up to $41 \%$ [2].

In the everyday dental practice, $\mathrm{SB}$ has been for many years commonly considered as a major risk factor for temporomandibular disorders (TMDs) [3]. TMD is a collective term embracing a number of clinical problems of the musculoskeletal structures of the masticatory system [4]. The most frequently

Konstantin Muzalev

k.muzalev@acta.nl

1 Department of Oral Kinesiology, Academic Centre for Dentistry

Amsterdam (ACTA), University of Amsterdam and Vrije

Universiteit Amsterdam, Gustav Mahlerlaan 3004, 1081, LA

Amsterdam, The Netherlands reported symptom of TMD is pain, which usually aggravates during masticatory function [5]. The origin of this pain most often lies to the masticatory muscles [6].

The assumption that SB is an important contributor for developing chronic jaw-muscle pain was tested in a number of studies that investigated a one-way relation between the oral parafunction and the TMD condition. Most of these studies had a cross-sectional design and led to contradictory conclusions regarding the association between $\mathrm{SB}$ and TMD pain $[7,8]$. Based on self-reports, bruxism has been found to be strongly associated with TMD pain.

In contrast, studies that used tooth wear as a manifestation of bruxism failed to prove an association with TMD pain $[9,10]$.

Currently, polysomnography (PSG) with simultaneous audio/video recording is considered the most accurate method to diagnose SB [11, 12]. Studies that used PSG for setting the diagnosis of SB yielded contradictory results regarding its association with TMD pain: some found a positive association [13], while others detected either no association [14] or even a negative one $[15,16]$.

These diverging results from studies on the SB-TMD association can, at least in part, be explained by the fact that 
previous studies dealt with patients with SB who suffered from TMD pain at the same time. Such a long-standing musculoskeletal pain, which fluctuates over time in presence and intensity, may have varying effects on jaw-muscle activity. There are two major but conflicting theories aiming to explain the association: the vicious cycle theory [17] and the pain adaptation model [18]. Nowadays, the pain adaptation model is considered to explain such effects best, suggesting that muscle pain leads to a decrease in muscle activity. However, there is still no common agreement on the effects of pain on muscle activity, leading to theories aiming to improve the extrapolation of these models (integrated pain adaptation model; [19]).

The aim of the present study was to assess how experimentally provoked TMD pain, caused by masticatory muscles' overloading, affects SB. We hypothesized that experimentally induced jaw-muscle pain would lead to decreasing jawmuscle activity during sleep.

\section{Materials and methods}

\section{Participants}

The following inclusion criteria were used to recruit study participants: self-report of presence of SB and presence of clinical signs of SB viz., muscle hypertrophy, hyperkeratosis of the tongue and cheek mucosa, tongue and lip impressions, tooth attrition, and/or matching wear facets on the teeth.

Participants were excluded when a shortened dental arch was present, because this would hinder wearing a protection splint during the pain provocation part of the protocol, when sleep disorders other than SB were present, when they used on a regular basis painkillers or medications that have a known influence on sleep structure or SB (e.g., selective serotonin reuptake inhibitors, anti-Parkinson medications), when TMD pain was present, when smoking more than five cigarettes per day or drinking more than two glasses of wine or equivalent alcoholic drinks per day, and/or when using a dental splint at night [20,21].

Participants were also excluded from the study in case they had less than 4 bruxism episodes per hour of sleep [11]. This exclusion parameter was assessed after the second ambulatory polysomnographic recording (see below). Patients were also excluded if no jaw-muscle pain was present $32 \mathrm{~h}$ after the experimental jaw-muscle pain provocation exercises.

To calculate sample size, "G power" software was used [22]. The following values to calculate sample size were used: Standard deviation for bruxism episodes per hour of sleep was 2 [23], the significance level was 0.05 , the power was 0.8 , and the clinically relevant difference was 3 episodes per hour of sleep. This resulted in a sample size of 10 .

In total, 23 male participants who self-reported sleep bruxism were screened. Twelve of them demonstrated clinical signs of sleep bruxism and agreed to participate in the study. The main reason for drop out at this stage was unwillingness to continue participation due to the complicated and timeconsuming design of the study.

Further, one participant was excluded after the second PSG recording due to too few (1.9) SB episodes per hour of sleep, and another two were excluded $32 \mathrm{~h}$ after the pain provocation test because no jaw muscle pain could be recorded.

Data from nine male volunteers (mean age \pm SD $29.2 \pm 5.7$; range 21-42 years) who fulfilled all inclusion criteria were used for the analysis. All participants were in good health. For all of them, the diagnosis of SB, graded as "probable," according to the diagnostic grading system for SB [1] was established. During the intake procedure, all participants were examined according to DC/TMD guidelines and no TMD pain diagnosis was established. The DC/TMD protocol was supplemented by dynamic and static pain tests [24]. These tests investigate possible pain from the masticatory muscles and/or temporomandibular joints by performing mandibular movements and static muscle efforts. During dynamic tests, the joint moves over its full range of motion against a slight manual resistance. During static tests, mandibular joints are held in place while the muscle exerts a high force. At baseline, all dynamic and static tests were negative.

The DC/TMD examination was performed by the same examiner (KM) for all participants. The examiner was extensively trained for DC/TMD protocol by a calibrated examiner (MK).

The study protocol was approved by the Medical Ethical Committee of the VU Medical Center (file number 2014/179). All subjects signed an informed consent form and received a non-pecuniary compensation (viz., an appointment for professional mouth cleaning and protection splint in case it was indicated) for their participation.

\section{Procedure}

At intake, an oral history was taken and a set of questionnaires was administered, including among others a standard Dutch medical questionnaire and the Dutch version of the Sleep Disorders Questionnaire (SDQ). In addition, a clinical examination was performed according to the DC/TMD protocol [25] and supplemented by dynamic and static pain provocation tests [24].

Following the intake procedure, the selected bruxers were invited to sleep with an ambulatory polysomnographic (PSG) device for two consecutive nights. Those of them who had at least 4 SB episodes per hour of sleep based on PSG analysis, underwent an experimental provocation protocol for the jaw muscles. This protocol has been proven to cause delayedonset muscle soreness (DOMS) with signs and symptoms leading to a TMD-pain diagnosis [26]. Thirty-two hours after the provocation part, participants were again examined 
clinically and a third PSG recording was made. These procedures are described below in detail.

\section{Polysomnography}

Ambulatory polysomnography (PSG) was performed using an Embla Titanium unit (Embla, Ontario, Canada) in the home environment of the participants for a total of three nights. The first two consecutive recordings were used to confirm the presence of SB and to document the characteristics of SB before the experimental TMD-pain provocation part (baseline). The third recording was performed $32 \mathrm{~h}$ after the provocation part to measure the SB when TMD-like pain was present.

The entire montage was performed in each participant's home. Participants were connected to the PSG device around 20:00 time. The recordings were made from approximately $22: 30$ to $7: 00$. Time of the recordings was closely adjusted to the participant's usual sleep time.

The electrodes for the PSG recordings were placed according to the protocol recommended by the American Academy of Sleep Medicine [27]. The montage protocol consisted of the following recordings:

1. Electroencephalography (EEG; F3, C4, O1, M1, M2)

2. Electro-oculography (EOG; right and left)

3. Electromyography (EMG; right masseter muscle, submental area)

The right masseter EMG signal was recorded at $512 \mathrm{~Hz}$ and filtered (Embla Titanium; $50 \mathrm{~Hz}$ notch; $3 \mathrm{~Hz}$ high pass; $100 \mathrm{~Hz}$ low pass). No audio/video recordings were obtained.

\section{Experimental TMD-pain provocation part}

The provocation of experimental TMD pain was performed $32 \mathrm{~h}$ before the third PSG registration using the protocol described extensively by Türker et al. [28]. In short, a "custommade" pain-provocation apparatus was used. Participants were sitting upright in a chair with an adjustable height, so that they could comfortably bite with their central incisors on the biting plates of the apparatus. During the provocation part, series of subsequent eccentric (opening while contracting the jaw closers) and concentric ("regular" closing) contractions of the jaw-closing muscles were performed in 12 sets of exercises, each lasting $5 \mathrm{~min}$ and with $1 \mathrm{~min}$ of rest in between. During the provocation part, the researcher repeatedly released the compression force of the apparatus, thus allowing the jaw-closing muscles to contract eccentrically.

During the provocation part, participants were constantly biting at a level of $25 \%$ of the baseline maximum voluntary contraction level of their right masseter muscle. The values of the EMG activity were displayed to the participant through visual feedback by the use of a voltmeter, with bipolar electrodes placed over the belly of the right masseter.

In order to protect the teeth from possible damage, participants were wearing soft acrylic mouth guards (Bioplast, $125 \mathrm{~mm}$, clear; Scheu Dental Technology, Iserlohn, Germany), made on individual plaster casts, on both dental arches throughout the entire provocation part.

\section{Pain measurement}

Participants were asked to rate the amount of jaw-muscle pain intensity on a questionnaire with a Numeric Rating Scale (NRS) every evening before the PSG recordings. Thus, a total of three NRS scores were obtained. Moreover, $32 \mathrm{~h}$ after the provocation part, a standardized clinical examination of the masticatory muscles and joints, according to the DC/TMD criteria supplemented by dynamic and static pain test, was performed in order to evaluate the presence of jaw-muscle pain (see above: procedure).

\section{Data analysis}

Before the PSG analysis, all registrations were coded to ensure that the examiner (KM) was blinded for the specific PSG recording under analysis.

Subsequently, all PSG recordings were analyzed with the use of RemLogic (Embla, Ontario, Canada) and Bruxism Detector (ACTA, Amsterdam, The Netherlands; custommade) software. The analyses consisted of two parts: a sleep analysis and a SB analysis. The sleep analysis was performed to exclude sleep disorders other than SB in addition to the SDQ (see above), to determine any abnormalities in sleep structure, and to enable the sole inclusion of masticatory muscle activities during actual sleep in the analysis of SB. Using 30 -s epochs, all sleep analyses were carried out automatically and checked manually according to the criteria described in The American Academy of Sleep Medicine Manual for the Scoring of Sleep [27]. Total sleep time and percentage of time spent in each sleep stage were calculated.

As a first step in the analysis of SB, the sleep stages and the EMG signal from the right masseter were transferred from RemLogic to Bruxism Detector software, using European Data Format (EDF). Bruxism Detector is a custom-made software that uses clinical research diagnostic criteria for SB (RDC/SB) proposed by Lavigne et al. [11] to quantify rhythmic masticatory muscle activity (RMMA) episodes. The EMG signal was low-pass and high-pass filtered $(100 \mathrm{~Hz}$ and $5 \mathrm{~Hz}$, respectively). During the next step, periods of increased EMG activity were detected using a threshold of three times above the noise level. During the last step, SB outcome variables, presented as the number of bruxism episodes per hour of sleep $\left(\mathrm{Epi} \mathrm{h}^{-1}\right)$, the number of bursts per hour of sleep $\left(\right.$ Bur h$^{-1}$ ), and the bruxism time index (BTI: the percentage of 
total sleep time spent bruxing) were calculated, and only the outcome variables detected during sleeping periods were used in the analyses. All analyses were manually checked.

\section{Statistics}

The non-parametric Friedman test was used to analyze the data. First, it was verified whether there were natural fluctuations in jaw muscle activity across the nights, using the SB data of the first two PSG recordings. Subsequently, it was tested whether there was a significant difference in SB activity between the night before and after the provocation part, comparing the data of the second and third PSG recordings. Statistical analysis was performed using IBM SPSS Statistics 23 software (IBM Corp., Armonk, NY, USA).

\section{Results}

Thirty-two hours after the DOMS provocation test, five participants reported pain graded as 1 on the 11 points NRS scale, three participants graded pain as 2, and one graded his pain as 8. For all of them, the diagnosis of myalgia could be established based on the DC/TMD protocol. They also responded positively with pain during both the dynamic and static tests. All nine participants described the pain during the test as mild and familiar to the pain they experienced after the provocation.

Based on the PSG recordings, all hypnograms (two baseline recordings and the recording $32 \mathrm{~h}$ after the jaw-muscle pain provocation) had a normal structure. Table 1 shows the mean values of the sleep variables.

The SB variables are presented in Table 2. The Friedman tests showed that there was no difference between the two PSG recordings made at baseline (first and second PSG recordings; $p=0.26-0.74)$. In contrast, after the pain provocation test (third PSG recording), a decrease for all SB parameters was recorded compared with the measurements of the second baseline PSG recordings $(p=0.01-0.03)$.

The decrease in SB activity varied among participants: for three of the participants, there was $<15 \%$ decrease in Epi $\mathrm{h}^{-1}$, for four participants, there was a decrease between $15 \%$ and $50 \%$, while for two participants, there was $>50 \%$ decrease (Figs. 1 and 5).

\section{Discussion}

The aim of the present study was to assess how experimental TMD pain, caused by masticatory muscles' overloading, affects SB. We hypothesized that experimentally induced jaw muscle pain would lead to decreasing jaw muscle activity during sleep. To that end, we provoked TMD-pain using a previously published protocol in pain-free bruxers and recorded the SB activity in the absence (before provocation) and presence (after provocation) of pain. Our results confirmed our research hypothesis, because a decline in SB activity was found for all nine participants who experienced jaw muscle pain after the pain provocation tests.

The effect of pain on jaw muscle activity has been a subject of interest in previous experimental studies as well. The results of these studies were contradictory: some showed an increase in muscle activity in response to pain [29], while others detected either no [30] or a negative effect of pain on jaw muscle activity [31]. These previous studies either used intramuscular injections (e.g., with capsaicin) to provoke the pain in humans [30] or were performed in animals [29]. To the best of our knowledge, this is the first study in humans on the effects of experimentally provoked TMD pain, due to muscle overloading, on SB.

The provocation protocol used in the present study has been previously described in detail and tested in a group of 40 healthy participants. It was shown that a state of DOMS can be provoked in healthy individuals, with signs and symptoms that lead to the diagnosis of TMD pain [26]. The advantage of this protocol, in contrast to the experimental models provoking short-lasting acute pain like the injection of painful substances into the muscles, is the fact that the pain is longer lasting and is present for several hours after the provocation part. Moreover, this type of experimental pain mimics the signs and symptoms of clinical TMD pain better, as compared to pain caused
Table 1 Descriptive statistics (mean \pm SEM) of the standard sleep variables of the two baseline nights and the night $36 \mathrm{~h}$ after experimental jaw-muscle pain provocation

\begin{tabular}{llllll}
\hline Sleep variable & Night 1 & Night 2 & Night 3 & P (N1-N2) & P (N2-N3) \\
\hline $\begin{array}{l}\text { Total sleep time (min) } \\
\text { Proportion of sleep (\%) }\end{array}$ & $456 \pm 25.7$ & $473 \pm 29.4$ & $443 \pm 21.2$ & 0.37 & 0.21 \\
Awake & $5.3 \pm 1.2$ & $3.3 \pm 1.9$ & $4.2 \pm 2.8$ & 0.58 & 0.44 \\
Stage 1 & $3.0 \pm 1.1$ & $5.9 \pm 3.1$ & $1.7 \pm 1.5$ & 0.61 & 0.93 \\
Stage 2 & $53.8 \pm 6.2$ & $56.1 \pm 4.9$ & $61.6 \pm 9.4$ & 0.18 & 0.59 \\
Stage 3 & $20.9 \pm 4.5$ & $18.5 \pm 1.4$ & $17.8 \pm 3.6$ & 0.32 & 0.27 \\
REM & $17.1 \pm 2.1$ & $16.1 \pm 4.4$ & $14.8 \pm 3.9$ & 0.76 & 0.82 \\
\hline
\end{tabular}


Table 2 Sleep bruxism variables (median; first and third quartiles, $p$ values based on Friedman test) of the two baseline nights and the night $36 \mathrm{~h}$ after experimental jaw muscle pain provocation

\begin{tabular}{llllll}
\hline Bruxism & Night 1 & Night 2 & Night 3 & P (N1-N2) & P (N2-N3) \\
\hline Epi h $^{-1}$ & $9.2(7,8-12,7)$ & $9.7(7.3-13.5)$ & $7.02(5.7-10.2)$ & 0.74 & 0.03 \\
Bur h $^{-1}$ & $66.7(47.7-79.9)$ & $75.2(45.1-99.5)$ & $51.1(36.5-70.1)$ & 0.32 & 0.01 \\
BTI & $2.2(1.6-2.4)$ & $2.0(1.4-2.7)$ & $1.7(0.9-2.1)$ & 0.48 & 0.01 \\
\hline
\end{tabular}

$E p i h^{-1}$ episodes per hour of sleep, Bur $h^{-1}$ bursts per hour of sleep, BTI Bruxism time index by other experimental protocols, like intramuscular injections. That is because the study protocol involves actual tissue damage due to overloading.

The way pain and muscle activity are related has been a matter of debate for several decades, leading to the publication of two major theories in order to explain this relation: the vicious cycle theory [17] and the pain adaptation model [18]. These two theories contradict each over. The vicious cycle theory suggests that an initiating factor, which could be SB for example, causes pain that reflexively leads to muscle spasm. This spasm leads to further pain and dysfunction, thus completing and perpetuating the loop. However, the evidence that supports the vicious cycle theory is until now limited $[32,33]$.

The pain adaptation model, on the other hand, suggests that muscle pain leads to a reduction in muscle activity of the painful muscles, aiming to protect the muscle system from further injury and, therefore, promote healing [34]. This model is commonly considered to explain most appropriately the effects of pain on muscle performance [19]. The reduced SB activity found after the experimental TMD pain was provoked and is in accordance with the Pain Adaptation Model. The pain causes a decrease in the muscle activity most probably in order to protect the muscles from further damage. This is in agreement with previous studies that found that in patients with TMD pain, the PSG-recorded bruxism is decreased compared with healthy controls [14].

The following possible limitations of this study should be kept in mind. First, even though we used a provocation protocol inducing longer-lasting TMD pain compared with other pain-provocation modalities, this pain still has an acute nature because it disappears after 1 week. TMD pain though is a chronic condition. It is likely that the relationship between chronic TMD pain and SB is more complex than just a linear association, and also that other factors, such as general health, genetics, and psychological status, may play an important role on the association under study [35].

Second, TMD pain has a higher prevalence in women [36]. In this study, we provoked jaw muscle pain in male volunteers in order to exclude the possible effects of cycle-dependent hormonal fluctuations on pain perception [37]. This limits the generalizability of our findings to the general TMD-pain population. It could also be interesting to investigate the possible gender differences in the association between TMD pain and SB, and whether hormones or other gender-related factors play a role in this association.

Third, even though we found a significant difference in SB activity before and after the provocation of experimental TMD pain on the group level, the individual participants reacted differently to the presence of pain. One participant showed a decrease of about $90 \%$ in SB activity, while another one demonstrated almost no changes in SB characteristics over the course of the three nights (see "Results" and Figs. 1 and 5). Considering an unusually low number of SB activity recorded for participant no. 3 (see Fig. 1, 2, 3, 4, and 5) during the third night registration, we checked whether his exclusion would provide a different study result. The difference between second- and third-night registrations still remained significant.

Hence, due to the high variation of the individual reactions to jaw muscle pain and the small size of the group, the extrapolation of our results to the population should be done with caution. Moreover, this variation could be an explanation why some patients develop chronic pain while others do not. One can speculate that a decrease of $90 \%$ in SB activity as a response to muscle damage leads to adequate protection from further injury and promotes healing. In contrast, it is unclear whether a decrease in muscle activity of, e.g., $15 \%$, is enough to protect damaged muscle tissue. It can be further speculated that some patients failed to "adjust" their jaw muscle activity to the amount that is needed for the recovery, and therefore damage the muscles. This phenomenon could be similar to the overtraining syndrome that is known to occur in limb muscles after excessive training.

Continuous low intensity load on the damaged limb muscle, such as walking, can cause a repetitive tissue trauma and lead to the development of chronic pain [38]. In our case, all participants were contacted 1 week after the provocation, and none of them reported any pain complaints. We also checked whether the variation in the decreased SB activity could be explained by the amount of pain that participants reported on NRS scale. The 
Fig. 1 Difference in SB activity measured by Epi $\mathrm{h}^{-1}$ between first and second nights (\%). *Epi $\mathrm{h}^{-1}$ - episodes per hour of sleep
Night 1 vs 2

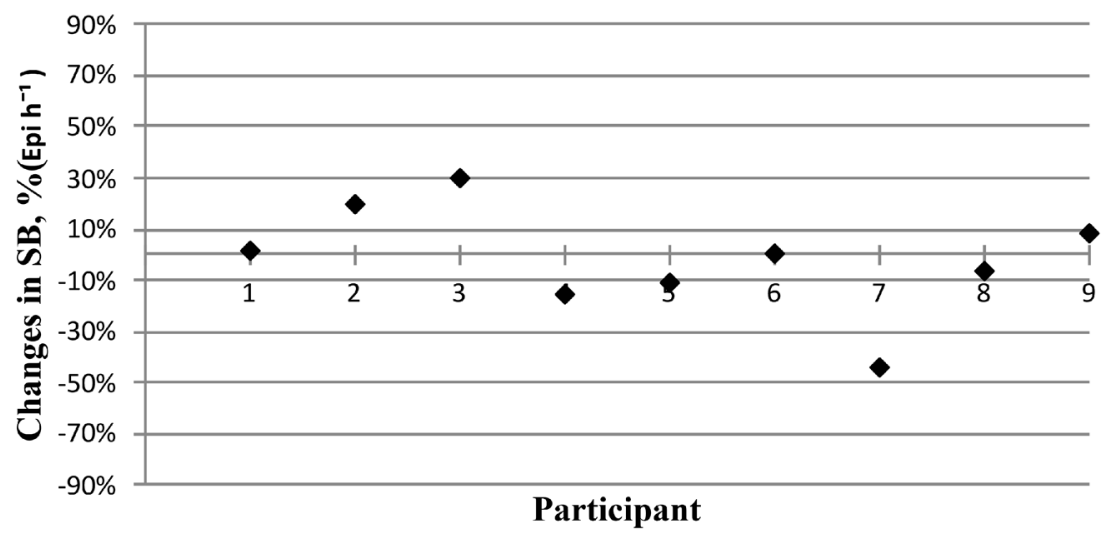

statistical analysis did not yield any significant results regarding a dose-response association $(\mathrm{r}=-.03, p=.92$, based on Spearman's correlation test).

Further, we recorded unusually high rates of SB episodes in our study (e.g., the average for the first two nights was nine SB episodes per hour of sleep). Previous studies that used sleep-laboratory environment with simultaneous audio/video registration demonstrated that the amount of sleep bruxism events per night is relatively low. For example, a previous study [39] showed that out of 146 participant who underwent two nights laboratory-based polysomnography registration, only 76 participants $(52 \%)$ had more than 1 SB episode per hour of sleep, and only 18 (13\% of the studied sample) had more than four SB episodes per hour of sleep, fulfilling the criteria for the high SB intensity group. Studies that compared the outcome of ambulatory polysomnography registrations with laboratory-based registrations have showed that the first one tends to overestimate SB [40]. With the absence of audio/video recordings, it is difficult to distinguish SB from other types of oromotor activities such as swallowing, lip sucking, chewing-like movements, etc. To counter the abovementioned disadvantage of ambulatory polysomnography recording, an additional registration channel can be used. For example, it has been documented that an increase in cardiac sympathetic activity ([41]) and an increase in breathing amplitude [42] precede SB. In future studies, registration of, e.g., heart rate, may improve the accuracy of scoring SB and may reduce false-positive outcomes of ambulatory PSG recordings. The diagnostic validity of these additional methods to diagnose SB has however yet to be verified.

Last but not least, the way the threshold for SB is defined could introduce a potential bias when patients with and without jaw muscle pain are compared. Previous study of Raphael et al. [43] suggested that the levels of EMG activity occurring outside SB and other nocturnal motor events are significantly higher in patients experienced TMD pain compared to painfree controls.

In fact, this background EMG activity is routinely considered as the threshold to define SB episodes. It
Fig. 2 Difference in SB activity measured by Epi $\mathrm{h}^{-1}$ between second and third nights (\%). *Epi $\mathrm{h}^{-1}$ - episodes per hour of sleep
Night 3 vs 2

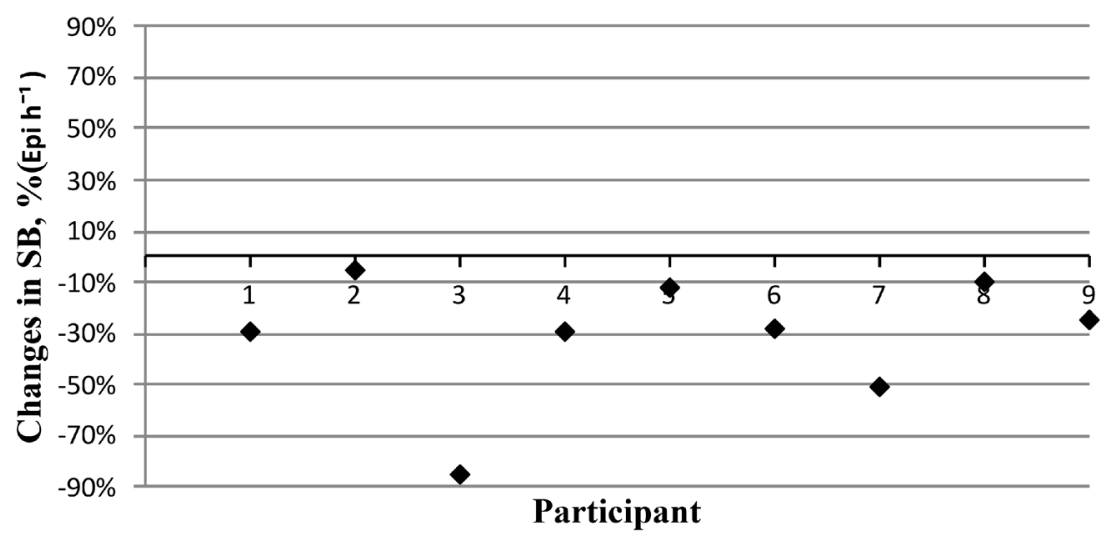


Fig. 3 Individual difference in SB episodes between first, second and third nights. *Epi $\mathrm{h}^{-1}$ episodes per hour of sleep

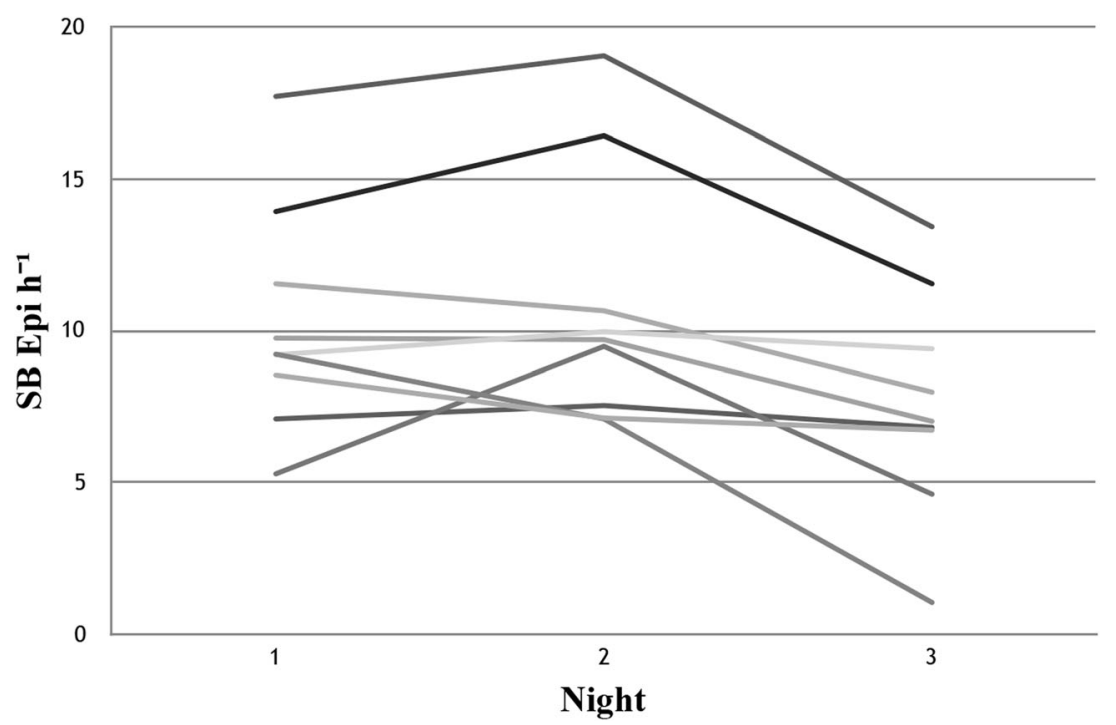

can be therefore speculated that the thresholds used to identify SB activity in this study were significantly higher after the pain provocation exercise when compared to two nights baseline registrations. These higher thresholds recorded in the presence of pain would univocally lead to inclusion less SB episodes during the third night registration when compared to two baseline registrations in the absence of jaw muscle pain. The alternative threshold could be the one based on percentages from the maximum voluntary contractions (MVCs), as proposed by Lavigne et al. [11].

However, using this threshold in the presence of jaw muscle pain could also introduce a bias. Participants could try to avoid pain during function and therefore do not express maximum bite force during the MVC registration at third night
PSG recording when jaw muscle pain was present. Therefore, there is a need for further investigation of the most reliable threshold for studies which aimed to compare the jaw muscle activity in TMD pain patients and pain-free individuals.

In conclusion, our study suggests that experimentally induced TMD pain reduces SB activity, which is in line with the pain adaptation model. It can be therefore expected that patients who experience TMD pain have less SB activity than pain-free individuals. Moreover, it can be assumed that eliminating jaw muscle pain during treatment could increase SB activity. Further research should take into account that the interaction between TMD pain and $\mathrm{SB}$ is complex, and caution in interpreting the results is required.
Fig. 4 Individual difference in SB bursts between first, second, and third night. * Bur $\mathrm{h}^{-1}$ _ bursts per hour of sleep

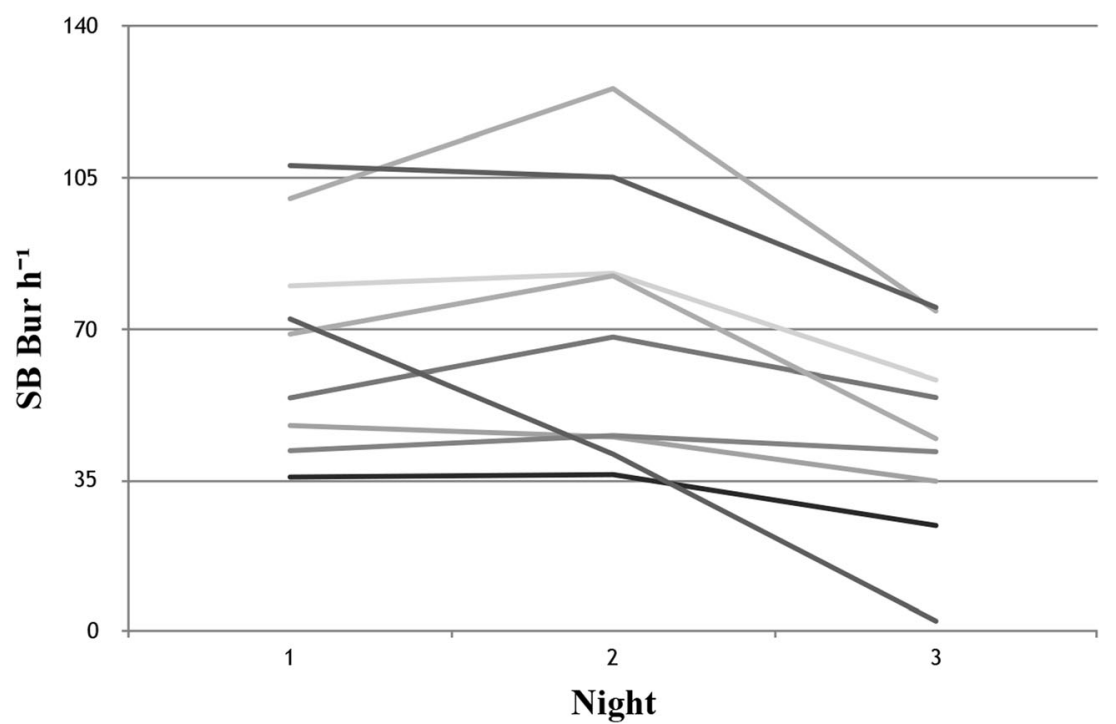


Fig. 5 Individual difference in SB time index between first, second, and third nights. *BTI-Bruxism time index

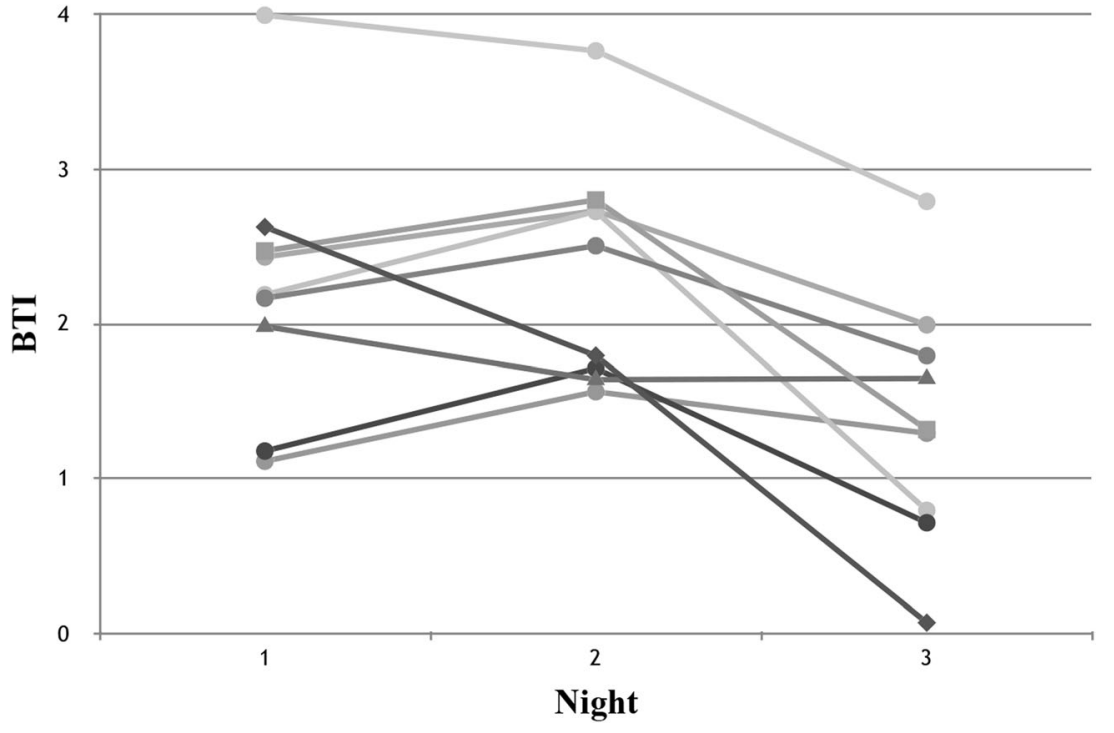

\section{Compliance with ethical standards}

Conflict of interest The authors declare that they have no conflict of interest.

Ethical approval All procedures performed in studies involving human participants were in accordance with the ethical standards of the institutional and/or national research committee and with the 1964 Helsinki declaration and its later amendments or comparable ethical standards.

Informed consent Informed consent was obtained from all individual participants included in the study.

Open Access This article is distributed under the terms of the Creative Commons Attribution 4.0 International License (http:// creativecommons.org/licenses/by/4.0/), which permits unrestricted use, distribution, and reproduction in any medium, provided you give appropriate credit to the original author(s) and the source, provide a link to the Creative Commons license, and indicate if changes were made.

\section{References}

1. Lobbezoo F, Ahlberg J, Glaros AG, Kato T, Koyano K, Lavigne GJ, de Leeuw R, Manfredini D, Svensson P, Winocur E (2013) Bruxism defined and graded: an international consensus. J Oral Rehabil 40(1):2-4

2. Manfredini D, Restrepo C, Diaz-Serrano K, Winocur E, Lobbezoo F (2013) Prevalence of sleep bruxism in children: a systematic review of the literature. J Oral Rehabil 40(8):631-642

3. Laskin DM (1969) Etiology of the pain-dysfunction syndrome. J Am Dent Assoc 79(1):147-153

4. McNeill C, Mohl ND, Rugh JD, Tanaka TT (1990) Temporomandibular disorders: diagnosis, management, education, and research. J Am Dent Assoc 120(3):253-257

5. Okeson JP (1996) Orofacial pain: guidelines for assessment, diagnosis, and management. Quintessence Publishing Company, Chicago
6. de Leeuw R, Klasser GD, Pain AAO (2013) Orofacial pain: guidelines for assessment, diagnosis, and management. Quintessence Publishing Company, Chicago

7. Lobbezoo F, Lavigne GJ (1997) Do bruxism and temporomandibular disorders have a cause-and-effect relationship? J Orofac Pain 11(1):15-23

8. Manfredini D, Lobbezoo F (2010) Relationship between bruxism and temporomandibular disorders: a systematic review of literature from 1998 to 2008. Oral Surg Oral Med Oral Pathol Oral Radiol Endod 109(6):26-50

9. Janal MN, Raphael KG, Klausner J, Teaford M (2007) The role of tooth-grinding in the maintenance of myofascial face pain: a test of alternate models. Pain Med 8(6):486-496

10. Schierz O, John MT, Schroeder E, Lobbezoo F (2007) Association between anterior tooth wear and temporomandibular disorder pain in a German population. J Prosthet Dent 97(5):305-309

11. Lavigne G, Rompre P, Montplaisir J (1996) Sleep bruxism: validity of clinical research diagnostic criteria in a controlled polysomnographic study. J Dent Res 75(1):546-552

12. Raphael K, Santiago V, Lobbezoo F (2016) Is bruxism a disorder or a behaviour? Rethinking the international consensus on defining and grading of bruxism. J Oral Rehabil 43(10):791-798

13. Rossetti LMN, Rossetti PHO, Conti PCR, CdRPd A (2008) Association between sleep bruxism and temporomandibular disorders: a polysomnographic pilot study. Cranio 26(1):16-24

14. Raphael KG, Sirois DA, Janal MN, Wigren PE, Dubrovsky B, Nemelivsky LV, Klausner JJ, Krieger AC, Lavigne GJ (2012) Sleep bruxism and myofascial temporomandibular disorders: a laboratory-based polysomnographic investigation. J Am Dent Assoc 143(11):1223-1231

15. Lavigne GJ, Rompre PH, Montplaisir JY, Lobbezoo F (1997) Short communication motor activity in sleep bruxism with concomitant jaw muscle pain. A retrospective pilot study. Eur J Oral Sci 105(1): 92-95

16. Rompré P, Daigle-Landry D, Guitard F, Montplaisir J, Lavigne G (2007) Identification of a sleep bruxism subgroup with a higher risk of pain. J Dent Res 86(9):837-842

17. Travell J, Rinzler S, Herman M (1942) Pain and disability of the shoulder and arm: treatment by intramuscular infiltration with procaine hydrochloride. JAMA 120(6):417-422

18. Lund JP, Donga R, Widmer CG, Stohler CS (1991) The painadaptation model: a discussion of the relationship between chronic 
musculoskeletal pain and motor activity. Can J Physiol Pharmacol 69(5):683-694

19. Murray GM, Peck CC (2007) Focus article: orofacial pain and jaw muscle activity: a new model. J Orofac Pain 21(4):279-288

20. Winocur E, Gavish A, Voikovitch M, Emodi-Perlman A, Eli I (2003) Drugs and bruxism: a critical review. J Orofac Pain 17(2): 99-111

21. Rintakoski K, Ahlberg J, Hublin C, Broms U, Madden P, Könönen M, Koskenvuo M, Lobbezoo F, Kaprio J (2010) Bruxism is associated with nicotine dependence: a nationwide Finnish twin cohort study. Nicotine Tob Res 12(12):1254-1260

22. Faul F, Erdfelder E, Lang A-G, Buchner A (2007) G*Power 3: a flexible statistical power analysis program for the social, behavioral, and biomedical sciences. Behav Res Methods 39(2):175-191

23. Van Der Zaag J, Lobbezoo F, Visscher CM, Hamburger HL, Naeije M (2008) Time-variant nature of sleep bruxism outcome variables using ambulatory polysomnography: implications for recognition and therapy evaluation. J Oral Rehabil 35(8):577-584

24. Visscher CM, Lobbezoo F, Naeije M (2007) A reliability study of dynamic and static pain tests in temporomandibular disorder patients. J Orofac Pain 21(1):39-45

25. Schiffman E, Ohrbach R, Truelove E, Look J, Anderson G, Goulet J-P, List T, Svensson P (2014) Diagnostic criteria for temporomandibular disorders (DC/TMD) for clinical and research applications: recommendations of the International RDC/TMD Consortium Network and Orofacial Pain Special Interest Group. J Orofac Pain 28(1):6-27

26. Koutris M, Lobbezoo F, Sümer NC, Atis ES, Türker KS, Naeije M (2013) Is myofascial pain in temporomandibular disorder patients a manifestation of delayed-onset muscle soreness? Clin J Pain 29(8): $712-716$

27. Iber C, Ancoli-Israel S, Chesson A, Quan S (2007) The AASM manual for the scoring of sleep and associated events American Academy of Sleep Medicine. American Academy of Sleep Medicine, Westchester

28. Türker KS, Koutris M, Sümer NC, Atıș ES, Linke IR, Lobbezoo F, Naeije M (2010) Provocation of delayed-onset muscle soreness in the human jaw-closing muscles. Arch Oral Biol 55(9):621-626

29. Ro JY, Svensson P, Capra N (2002) Effects of experimental muscle pain on electromyographic activity of masticatory muscles in the rat. Muscle Nerve 25(4):576-584

30. Arima T, Arendt-Nielsen L, Svensson P (2001) Effect of jaw muscle pain and soreness evoked by capsaicin before sleep on orofacial motor activity during sleep. J Orofac Pain 15(3):245-256

31. Svensson P, Arendt-Nielsen L, Houe L (1996) Sensory-motor interactions of human experimental unilateral jaw muscle pain: a quantitative analysis. Pain 64(2):241-249
32. Stohler CS, Zhang X, Lund JP (1996) The effect of experimental jaw muscle pain on postural muscle activity. Pain 66(2):215-221

33. Svensson P, De Laat A, Graven-Nielsen T, Arendt-Nielsen L (1998) Experimental jaw-muscle pain does not change heteronymous $\mathrm{H}$ reflexes in the human temporalis muscle. Exp Brain Res 121(3): 311-318

34. Lund JP, Lavigne GJ, Dubner R, Sessle BJ (2001) Orofacial pain: from basic science to clinical management. Quintessence, Chicago

35. Slade G, Ohrbach R, Greenspan J, Fillingim R, Bair E, Sanders A, Dubner R, Diatchenko L, Meloto C, Smith S (2016) Painful temporomandibular disorder: decade of discovery from OPPERA studies. J Dent Res 95(10):1084-1092

36. LeResche L (1997) Epidemiology of temporomandibular disorders: implications for the investigation of etiologic factors. Crit Rev Oral Biol Med 8(3):291-305

37. Thibodeau MA, Welch PG, Katz J, Asmundson GJ (2013) Painrelated anxiety influences pain perception differently in men and women: a quantitative sensory test across thermal pain modalities. Pain 154(3):419-426

38. Stauber WT (2004) Factors involved in strain-induced injury in skeletal muscles and outcomes of prolonged exposures. J Electromyogr Kinesiol 14(1):61-70

39. Muzalev K, Lobbezoo F, Janal M, Raphael K (2017) Inter-episode sleep bruxism intervals and myofascial face pain. Sleep 40(8). doi: https://doi.org/10.1093/sleep/zsx078

40. Gallo LM, Lavigne G, Rompré P, Palla S (1997) Reliability of scoring EMG orofacial events: polysomnography compared with ambulatory recordings. J Sleep Res 6(4):259-263

41. Huynh N, Kato T, Rompré PH, Okura K, Saber M, Lanfranchi PA, Montplaisir JY, Lavigne GJ (2006) Sleep bruxism is associated to micro-arousals and an increase in cardiac sympathetic activity. J Sleep Res 15(3):339-346

42. Khoury S, Rouleau GA, Rompré PH, Mayer P, Montplaisir JY, Lavigne GJ (2008) A significant increase in breathing amplitude precedes sleep bruxism. CHEST 134(2):332-337

43. Raphael KG, Janal MN, Sirois DA, Dubrovsky B, Wigren PE, Klausner JJ, Krieger AC, Lavigne GJ (2013) Masticatory muscle sleep background electromyographic activity is elevated in myofascial temporomandibular disorder patients. J Oral Rehabil 40(12):883-891

Publisher's note Springer Nature remains neutral with regard to jurisdictional claims in published maps and institutional affiliations. 